\title{
Un estudio exploratorio sobre la donación hipotética de embriones humanos en el Perú *
}

\author{
An exploratory study regarding the hypothetical human embryo donation in Peru
}

\author{
Jorge Alberto Álvarez-Díaz ${ }^{1}$ \\ * Trabajo presentado en parte en el VIII Congreso Nacional de Bioética de la Asociación de Bioética Fundamental y Clínica (ABFyC), Oviedo, España, 2007. \\ ${ }^{1}$ Médico sexólogo clínico, master en bioética. Doctorando en el Programa de Ciencias Sociosanitarias y Humanidades Médicas, Universidad Complutense de Madrid.
}

\begin{abstract}
Resumen
Objetivo: Explorar opiniones de pacientes que han acudido a técnicas de reproducción asistida (TRA) complejas, respecto a la donación de gametos y embriones, así como las razones para hacerlo o no. Diseño: Estudio transversal de bioética descriptiva, con metodología cualitativa etnográfica, mediante entrevista semiestructurada, aplicando análisis del discurso al texto resultante. Lugar: Clínica privada, en Lima, Perú. Participantes: Veinte mujeres y 12 hombres, quienes habían acudido por lo menos a una TRA compleja. Intervenciones: Entrevista semiestructurada, aplicando análisis del discurso al texto resultante. Principales medidas de resultados: Opinión sobre donación de gametos y embriones. Resultados: Respecto a la donación de embriones, 11 de los hombres los donarían con fines de terapia de fertilidad, 6 con fines de investigación y solamente uno rechazó ambas posibilidades de donación. Las 20 mujeres entrevistadas, por su parte, los donarían para terapia de fertilidad y 8 de ellas con fines de investigación. Los participantes que han aceptado gametos donados, no necesariamente piensan que donarían los propios en caso de poder hacerlo. Conclusiones: La donación de gametos es más comentada y generalmente aceptada; la donación de embriones es un tema menos discutido y más conflictivo, tanto para donar como para aceptar. La criopreservación es un tema complejo, comentado pero también conflictivo, cuya aceptación o no, así como el destino de los embriones probablemente criopreservados, depende de las concepciones que se tiene respecto al origen de la vida, la ética personal, entre otros. Se puede plantear una hipótesis, a ser verificada en estudios cuantitativos, de que la donación de embriones podría efectuarse, principalmente, para terapia de fertilidad y excepcionalmente con fines de investigación.

Palabras clave: Destinación del embrión; criopreservación; técnicas reproductivas asistidas; fertilización in vitro; inyecciones de esperma intracitoplasmáticas; células germinativas; Perú.
\end{abstract}

\begin{abstract}
Objective: To explore patients undergoing complex assisted reproductive technologies' (ART) opinions on both gamete and embryo donation, as well as the reasons to do it or not. Design: Cross-sectional study of descriptive bioethics, with ethnographic qualitative methodology using a semi-structured interview, applying speech analysis to the resulting written transcript. Setting: Private medical institution, in Lima, Peru. Participants: Twenty women and 12 men who had had at least one complex ART. Interventions: Semi-structured interview, applying speech analysis to the resulting written transcript. Main outcome measures: Opinion on both gamete and embryo donation. Results: Regarding embryo donation, 11 men would donate their embryos for infertility treatment, 6 for research purposes, and only one rejected both possibilities. On the other hand, all 20 women would donate embryos for infertility treatment and only 8 for research purposes. Participants who accepted gamete donations did not necessarily think they would donate their own gametes. Conclusions: Results suggest gamete donation is more commented and generally accepted; embryo donation, either to donate or to accept donation, is a more conflicting and less discussed subject. Cryopreservation is a complex subject, commented but also conflicting, whose acceptance or not as well as the probable cryopreserved embryos destiny depend on participants beliefs on life origin, personal ethics, etc. Hypothesis resulting from this study to be verified in future quantitative researches is that embryo donation could take place mainly for infertility treatment and exceptionally for research.

Key words: Embryo disposition; cryopreservation; reproductive techniques, assisted; fertilization in vitro; sperm injections, intracytoplasmic; germ cells, Peru.
\end{abstract}

\section{INTRODUCCIÓN}

Esterilidad es la incapacidad del varón o de la mujer de concebir ${ }^{(1)}$; se la clasifica en primaria y secundaria. Infertilidad es la incapacidad para producir un hijo vivo, y también es clasificada en primaria y secundaria. Como el término 'infertilidad' habla de 'incapacidad', en clínica médica sería más propio el término de 'subfertilidad'. Este grupo de la población se ha beneficiado de los tratamientos con técnicas de reproducción asistida (TRA), que pueden ser simples (como la inseminación intrauterina) o complejas (como la fecundación in vitro con transferencia de embriones -FIV - o la inyección intracitoplásmica del esperma -ICSI). Para el caso peruano, los antecedentes de TRA simples muestran que, en caso de factor cervical o en factor masculino con astenozoospermia, la inseminación con semen del esposo tiene una tasa de éxito aceptable ${ }^{(2)}$, pero menor que en pacientes con azoospermia que acuden a inseminación con semen de donador ${ }^{(3)}$.

La primera técnica de reproducción asistida compleja, la FIV, fue desarrollada por Steptoe y Edwards, en Inglaterra, culminando con el nacimiento de Louise Joy Brown, en $1978^{(4)}$. En América Latina, es Chile que comunicó el primer nacimiento de un producto conseguido mediante FIV, en $1984^{(5)}$. Perú cuenta también con experiencia en TRA complejas, como lo muestran el Registro Latinoamericano de Reproducción Asistida (RLA) de la Red Latino Americana de Reproducción
Asistida (Red LARA), en los últimos años $(\text { Tabla } 1)^{(6)}$.

Por otro lado, en 1983, Trounson consiguió en Australia el primer nacimiento resultado del embarazo con un embrión criopreservado ${ }^{(7)}$. Esto cambió de manera importante las TRA complejas, ya que así existía la posibilidad de que la mujer no se sometiera a todo el tratamiento completo, puesto que bastaba que se presentara a la transferencia embrionaria. La criopreservación de embriones suponía un ahorro económico y de estrés (físico y psicológico), al evitar la hiperestimulación ovárica, así como ahorro en los medios de cultivo para que se realizara la FIV; se evitaba estrés al varón en la colecta de la muestra, entre otros. Esto ha tenido repercusiones desta- 
Tabla 1. Comunicación sobre ciclos iniciados de reproducción asistida en el Perú, informados a la Red Latino Americana de Reproducción Asistida, en el periodo 1999-2005.

\begin{tabular}{ccccc}
\hline Año & $\begin{array}{c}\mathrm{N}^{\circ} \text { de centros } \\
\text { que informaron }\end{array}$ & $\begin{array}{c}\mathrm{N}^{\circ} \text { de ciclos } \\
\text { iniciados }\end{array}$ & $\begin{array}{c}\mathrm{N}^{\circ} \text { promedio de ciclos iniciados } \\
\text { mensualmente por centro }\end{array}$ & $\begin{array}{c}\text { \% del total latinoamericano } \\
\text { de ciclos reportados a la Red }\end{array}$ \\
\hline 1999 & 2 & 314 & 13,1 & 2,1 \\
2000 & 2 & 397 & 16,5 & 2,5 \\
2001 & 2 & 458 & 19,1 & 2,5 \\
2002 & 2 & 544 & 22,7 & 2,9 \\
2003 & 1 & 455 & 37,9 & 2,1 \\
2004 & 2 & 1047 & 43,6 & 4,3 \\
2005 & 2 & 1169 & 48,7 & 4,4 \\
\hline
\end{tabular}

cables en la criobiología humana, donde existe la posibilidad de criopreservar espermatozoides, ovocitos, pronúcleos, embriones desde 2 células hasta blastocistos, tejido ovárico y testicular. Pero, también el contar con la posibilidad de mantener un embrión criopreservado genera un replanteamiento de muchos problemas importantes: teológicos, tales como la consideración de la animación ${ }^{(8)}$, y bioéticos, como la elección del destino de los embriones supernumerarios o la posibilidad de poder realizar investigación en embriones humanos ${ }^{(9)}$.

Por todas las consideraciones previas es que en la actualidad la donación de embriones es una realidad en países desarrollados. Las sociedades que agrupan profesionales de la medicina reproductiva han propuesto guías y recomendaciones éticas básicas a los centros que llevan a cabo los procedimientos, tanto en EE UU, a través de la American Society for Reproductive Medicine (ASRM) ${ }^{(10)}$, como en Europa, a través de la European Society for Human Reproduction and Embryology (ESHRE) ${ }^{(11)}$.

A partir de la nueva realidad de combinar FIV con criopreservación, aparece un nuevo problema: ¿qué hacer con los embriones que están criopreservados? Se resume las posibilidades en tres: que sigan criopreservados hasta que las parejas decidan utilizarlos para sí mismas con fines reproductivos; que decidan donarlos (con fines reproductivos, de investigación o terapéuticos) o que decidan descartarlos (o desecharlos). De esta manera es que se ha iniciado los estudios, básicamente ante la realidad que presentan las naciones desarrolladas: parejas que tratan su incapacidad de reproducir con embriones criopreservados.

La realidad latinoamericana es distinta por muchos factores: económicos, culturales, religiosos, sociales, entre otros ${ }^{(12)}$. Históricamente, las TRA y sus avances llegaron en fechas posteriores a América Latina; no es accesible a toda pareja el pagar un ciclo para una TRA o la criopreservación de embriones (actividades costeadas principalmente en centros privados); los programas de donación son un tanto distintos (prácticamente no hay programas de donación de embriones, las donadoras puras de ovocitos no son la constante, entre otros); el cristianismo es predominante (de este, la forma más común es el catolicismo, con más de $80 \%$ de la población total) y su influencia tiene aún un peso importante (directa o indirectamente) en las decisiones personales de muchas parejas.

Se ha propuesto que, de no considerar esta reflexión, tanto desde el punto de vista bioético como biojurídico, América Latina podría convertirse tan solo en un mercado para naciones desarrolladas de venta de gametos y de compra de embriones. Son escasas las naciones que cuentan con legislaciones que esbozan el tema específico de la reproducción asistida o la investigación con embriones humanos, así que los marcos legales son casi inexistentes.

Todo lo expuesto hasta el momento explica, al menos en parte, que no existan a la fecha trabajos que expongan si las parejas latinoamericanas donarían sus embriones (y bajo qué circunstancias) o no. La temática ya de por sí es bastante compleja.

El objetivo del presente trabajo es explorar opiniones de pacientes que han acudido a TRA complejas (FIV, ICSI y otros), en el Perú, respecto a la donación hipotética de embriones, así como las razones para hacerlo o no, con la idea de generar hipótesis para estudios posteriores, de corte cuantitativo.

\section{MÉTODOS}

Se realizó un estudio transversal de bioética descriptiva. La metodología fue cualitativa etnográfica. El instrumento utilizado fue una entrevista oral semiestructurada individual, grabada y transcrita, aplicando la técnica de análisis del discurso al texto resultante.

Originalmente, se pensó en que participaran los dos centros en Lima que comunican su actividad a la Red LARA; pero, de ambos centros solamente aceptó colaborar uno de ellos. Por ello, el proyecto se desarrolló solamente en una clínica privada de Lima, Perú
(Concebir, del Grupo Pranor), contando con la aprobación de su Comité de Ética y respetando la normativa nacional peruana en materia de investigación, así como la normativa internacional (Helsinki y las Pautas Éticas para la Investigación en Seres Humanos, del Consejo de Organizaciones Internacionales de las Ciencias Médicas (CIOMS), dentro del marco del Programa Internacional de Formación en Ética de la Investigación Biomédica y Psicosocial. La muestra seleccionada fue no probabilística. Los criterios de inclusión fueron parejas cuyo diagnóstico de subfertilidad les hubiera hecho tributarias de tratamiento con TRA complejas, haber acudido al menos a un ciclo iniciado de tratamiento y asistir a la clínica para responder una entrevista. No hubo criterios de exclusión.

En una primera fase, para el proceso del consentimiento informado se envió una carta a los pacientes que habían participado en una TRA, como sensibilización y primera invitación. Posteriormente, se realizó una llamada telefónica, para reforzar la invitación y darles las generalidades de la investigación. En esta primera fase, la información era proporcionada por la licenciada en obstetricia, coordinadora del Programa.

En una segunda fase del proceso del consentimiento, los pacientes que aceptaron acudir a la clínica recibieron una explicación detallada por parte del investigador y, si aceptaban, firmaban un formato para evidenciar el proceso. Se ofrecía la opción de responder la entrevista en ese momento o dar más tiempo, en caso de requerirlo. En todos los casos, los participantes respondieron tras la firma del formato del consentimiento informado. Para realizar la entrevista, se realizó una estancia por parte del investigador, de enero a marzo de 2005. Se acudía a diario al centro, donde se facilitó un consultorio, para realizar las entrevistas, con las condiciones adecuadas de luminosidad, privacidad y otros. Se invitó a un total de 100 personas, aceptando participar finalmente solo 32 .

La entrevista se realizó siempre de forma individual, se grabó en un medio electrónico, se transcribió textualmente y al trascrito resultante se aplicó la metodología de análisis del discurso. En el análisis, se buscó intencionadamente los argumentos a favor o en contra de la donación.

\section{RESULTADOS}

Se entrevistó a 20 mujeres y 12 varones, lo que revela que no siempre fue posible entrevistar al cónyuge respectivo (Tabla 2). 
Tabla 2. Caracteristicas médicas y sociodemográficas de los participantes ( $1^{\mathrm{a}}$ parte).

\begin{tabular}{|c|c|c|c|c|c|c|c|c|}
\hline Entrevistado & Género & Edad & $\begin{array}{l}\text { Años de } \\
\text { esterilidad }\end{array}$ & TRA & $\begin{array}{l}\text { Número de } \\
\text { procedimientos }\end{array}$ & Hijos nacidos vivos & $\begin{array}{l}\text { Años de } \\
\text { escolaridad }\end{array}$ & Religión \\
\hline 1 & $\mathrm{~F}$ & 39 & 4 & FIV & 1 & No & 16 & Católica \\
\hline 2 & $\mathrm{~F}$ & 42 & 3 & ICSI & 2 & No & 15 & Católica \\
\hline 3 & $\mathrm{~F}$ & 33 & 3 & ICSI & 3 & No & 15 & Católica \\
\hline 4 & $\mathrm{~F}$ & 39 & 8 & FIV + ovodonación & 1 & No & 14 & Católica \\
\hline 5 & M & 34 & 8 & FIV + ovodonación & 1 & No & 11 & Católica \\
\hline 6 & $\mathrm{~F}$ & 41 & 5 & FIV & 1 & $\begin{array}{l}\text { No (Con un embarazo gemelar de } 20 \text { semanas } \\
\text { al momento de la entrevista) }\end{array}$ & 16 & Católica \\
\hline 7 & M & 45 & 7 & FIV & 1 & No & 11 & Católica \\
\hline 8 & $\mathrm{~F}$ & 40 & 7 & FIV & 1 & No & 14 & Católica \\
\hline 9 & $\mathrm{~F}$ & 35 & 9 & FIV, FIV + ovodonación & $5(4$ y 1$)$ & No & 14 & Católica \\
\hline 10 & M & 42 & 3 & FIV & 1 & No & 18 & Católica \\
\hline 11 & M & 38 & 5 & FIV & 3 & No & 11 & Católica \\
\hline 12 & $\mathrm{~F}$ & 41 & 5 & FIV & 3 & No & 16 & Católica \\
\hline 13 & M & 40 & 15 & FIV + ovodonación & 1 & No & 9 & Católica \\
\hline 14 & $\mathrm{~F}$ & 32 & 15 & FIV + ovodonación & 1 & No & 6 & Católica \\
\hline 15 & $\mathrm{~F}$ & 33 & 3 & FIV & 3 & No & 16 & Católica \\
\hline 16 & M & 45 & 9 & ICSI & 1 & No & 11 & Católica \\
\hline 17 & $\mathrm{~F}$ & 28 & 9 & ICSI & 1 & No & 14 & Católica \\
\hline 18 & M & 43 & 6 & FIV & 2 & No & 16 & Católica \\
\hline 19 & $\mathrm{~F}$ & 39 & 6 & FIV & 2 & No & 16 & Católica \\
\hline 20 & $F$ & 34 & 1 & FIV & 1 & $\begin{array}{l}\text { Si (Un nacido vivo obtenido por operación } \\
\text { cesárea, el día anterior a la entrevista) }\end{array}$ & 14 & Católica \\
\hline
\end{tabular}

El rango de las edades fue 28 a 47 años, con una media de 36,9 $\pm 5,2$ años. Los años de escolaridad fueron en promedio $14,1 \pm 2,6$ (con un amplio rango de 6 a 18, colocando en ambos extremos a una entrevistada con educación básica y, en el otro extremo, un entrevistado con maestría). El 96,9\% se refería a sí mismo como de religión católica y 3,1\%, como evangélico, lo que representa que $100 \%$ de la muestra entrevistada se consideró creyente y practicante de religiones con orientación cristiana. Todos eran ciudadanos peruanos, la mayoría de ellos de Lima y cercanías.

El tiempo de esterilidad fue muy variable, de uno a 17 años, con una media de 6,3 $\pm 3,8$ años. De acuerdo a la técnica de fecundación asistida a la cual fueron sometidos, $75 \%$ de los entrevistados tuvo FIV (de los cuales, 54,2\%, FIV con ambos gametos propios, 20,8\% con ovodonación, $8,3 \%$ con semen de donador y $16,7 \%$ con gestación surrogada), mientras que $25 \%$ tuvo ICSI. El número de procedimientos a los cuales se habían sometido, en promedio, fue de $1,7 \pm 1,1$. El 3,1\% de los entrevistados tenía un hijo nacido vivo (con una pareja anterior), 6,3\% tenía un hijo nacido vivo de ambos (logrado por FIV y obtenido mediante operación cesárea un día antes de la entrevista), mientras que 90,6\% no había tenido hijo nacido vivo.

Respecto a donar o recibir gametos donados, las respuestas fueron muy consistentes entre los hombres, ya que 10 de ellos aceptarían donar tanto espermatozoides como ovocitos a otra pareja y solo dos de ellos no. Por su parte, 15 de las mujeres aceptarían que su pareja donara espermatozoides (5 no lo harían) y este número aumenta a 17 si se trata de la posibilidad de donación de los ovocitos propios (y solamente 3 no lo harían). Respecto a aceptar gametos de un tercero, en el tema de los espermatozoides,

Tabla 3. Participantes de acuerdo a la aceptación en la donación y recepción de gametos.

\begin{tabular}{|c|c|c|c|c|c|c|c|c|}
\hline & \multicolumn{4}{|c|}{ Donación de gametos } & \multicolumn{4}{|c|}{ Recepción de gametos } \\
\hline & \multicolumn{2}{|c|}{ Espermatozoides } & \multicolumn{2}{|c|}{ Óvulos } & \multicolumn{2}{|c|}{ Espermatozoides } & \multicolumn{2}{|c|}{ Óvulos } \\
\hline & Aceptación & Rechazo & Aceptación & Rechazo & Aceptación & Rechazo & Aceptación & Rechazo \\
\hline Masculinos & 10 & 2 & 10 & 2 & 9 & 3 & 10 & 2 \\
\hline Femeninos & 15 & 5 & 17 & 3 & 17 & 3 & 18 & 2 \\
\hline
\end{tabular}

Tabla 4. Participantes de acuerdo a la aceptación en la donación y recepción de embriones.

\begin{tabular}{lccccccc}
\hline & \multicolumn{3}{c}{ Donación de embriones } & & \multicolumn{3}{c}{ Recepción de embriones } \\
\cline { 2 - 3 } & $\begin{array}{c}\text { Terapia de } \\
\text { fertilidad }\end{array}$ & Investigación & Rechazo & & Aceptación & $\begin{array}{c}\text { Aceptación con } \\
\text { restricciones }\end{array}$ & Rechazo \\
\hline Masculinos & 11 & 6 & 1 & & 0 & 11 & 1 \\
Femeninos & 20 & 8 & 0 & & 0 & 18 & 2 \\
\hline
\end{tabular}

9 hombres lo aceptarían, mientras que tres no; para el caso de que estos varones consideraran que su pareja aceptara ovocitos, 10 lo aceptaría y 2 de ellos no. Las mujeres, por su parte, en el tema de la donación de gametos, 15 de ellas aceptarían que su pareja donara semen ( 5 de ellas no), mientras que 17 de ellas aceptaría donar sus ovocitos (y solamente 3 de ellas no). Las mujeres, en el tema de la aceptación de gametos de un donante, 17 estarían de acuerdo en recibir espermatozoides y 18 de ellas, ovocitos (Tabla 3).

El tema se hace más complejo respecto a la posibilidad de donación embrionaria. Esto, por varios motivos; uno de ellos fue la falta de información y que varios participantes no habían siquiera pensado en la posibilidad de donar o recibir un embrión de otra pareja. Respecto a la posibilidad de donar embriones propios, 11 de los hombres pensaba que lo haría para terapia de fertilidad, 6 para realizar investigación y 1 definitivamente descartaría ambas posibilidades; la suma no es equivalente a los 12 participantes, debido a que las posibilidades de donación, al ser hipotéticas, no eran mutuamente excluyentes. Por su parte, la totalidad de las mujeres pensaba que donaría sus embriones para terapia de fertilidad y 8 consideraba que donaría con fines de investigación. Respecto a aceptar un embrión donado, en principio ningún hombre o mujer lo aceptaría completamente sin restricciones; 11 de los hombres lo aceptaría con alguna restricción y solo 1 lo rechazaría, mientras que 18 mujeres lo aceptaría y 2 lo rechazaría (Tabla 4). 


\section{DISCUSIÓN}

Un estudio de 1992, en EE UU, mostró que, de un grupo de 365 pacientes, $44 \%$ preferiría continuar con la criopreservación para uso propio más adelante, $43 \%$ pensaba descartarlos y solo $13 \%$ optaría por la donación (11\% a parejas infértiles y 10\% para investigación) ${ }^{(13)}$. Un estudio posterior realizó un envío de 2210 encuestas, siendo regresadas 1244 (63\% de ellas respondidas por mujeres), 1020 con embriones criopreservados; $49 \%$ de los últimos opinaba que donaría sus embriones con fines de investigación (lo que representaría el destino de 2000 a 3050 embriones), mientras que quienes donarían sus embriones con fines de terapia de fertilidad no alcanzaban el 20\% ${ }^{(14)}$.

En Australia, se ha realizado varios estudios respecto a opiniones sobre la donación de embriones. Uno con 1246 parejas mostró que $89,5 \%$ preferiría descartar que donar sus embriones ${ }^{(15)}$. En otro estudio con 509 parejas, solo $10 \%$ consideraba probable y $34 \%$ posible la donación para investigaciones médicas; las respuestas negativas fueron más frecuentes entre mujeres que habían tenido los embriones criopreservados por más tiempo y quienes manifestaban la práctica de alguna religión, argumentando que consideraban al embrión como un niño potencial y que podría perderse el control sobre las investigaciones médicas; las respuestas positivas comentaban que no querían desperdiciar embriones, que querían ayudar a parejas subfértiles o que querían contribuir al avance del conocimiento científico ${ }^{(16)}$. Otro estudio mostró que, de un grupo de 126 pacientes, un 30\% los donaría para investigación ${ }^{(17)}$. Un estudio posterior muestra que $90 \%$ de los pacientes optó por descartar sus embriones, en lugar de donarlos ${ }^{(18)}$; aunque, en otro realizado en 311 parejas, $42 \%$ aceptaría la donación de embriones para investigación ${ }^{(19)}$.

Al recabar la experiencia italiana, de 10 años, se muestra que solamente $6 \%$ de los pacientes permitiría la donación de sus embriones ${ }^{(20)}$. En Dinamarca, un estudio en 207 parejas mostró que, con resultados que no son mutuamente excluyentes, $28,5 \%$ donaría sus embriones a una pareja subfértil, $60,4 \%$ para investigación en infertilidad, $56,5 \%$ para investigación en células troncales y $48,8 \%$ para tratamiento con células troncales ${ }^{(21)}$.

Con estos datos, se puede notar algo en común: en naciones desarrolladas parece ser más sencillo descartar los embriones supernumerarios o, en su caso, donarlos con fines de investigación, que donarlos para terapia de fertilidad. Esto llama la atención, porque en definitiva no coincide con lo encontrado en el estudio cualitativo en una nación menos desarrollada, como es el caso peruano.

Posteriormente, se inició estudios para evaluar factores de predictibilidad para la donación de embriones, aunque no se tuviera ya criopreservados. Un estudio canadiense en 49 parejas mostró que la comodidad respecto a compartir información con la pareja que recibiría el embrión era más importante que los procedimientos de tamizaje para la aceptación de la donación (22). Este aspecto no coincide con lo encontrado entre los participantes de nuestro estudio, ya que los comentarios que tenían respecto eran precisamente en la línea de asegurase que el embrión estuviese sano y, secundariamente, con algunos rasgos fenotípicos, en la medida que pudiesen ser similar a los padres; así, una mujer comentaba "Me gustaría saber las características físicas para aceptar un embrión donado".

Aunque no es frecuente la utilización de metodología cualitativa, también el campo de la medicina reproductiva ha notado la deficiencia de este tipo de estudios, por lo que han aparecido cada vez más con este enfoque, explorando temas, como la vivencia de la subfertilidad ${ }^{(23)}$ o de las TRA ${ }^{(24)}$. Al realizar estudios cualitativos respecto a la donación de embriones, en EE UU se ha hecho notar que no se ha explorado las actitudes del tipo de participantes incluidos en la investigación (25). Otro estudio cualitativo realizado en EE UU se refiere a las conceptualizaciones que tienen los padres respecto a los embriones (26), las cuales varían desde considerar a los embriones criopreservados como tejido biológico, entidades vivientes, 'niños virtuales' con intereses que deben ser considerados y protegidos, hermanos de los hijos nacidos, hasta recuerdos simbólicos de su subfertilidad pasada. Para el presente estudio, ninguno de los participantes consideró a sus embriones como tejido biológico ni como recuerdo de su subfertilidad. La mayoría consideró que los embriones tenían más relación ontológica con un ser humano equiparable a un hijo nacido vivo, con expresiones tales como "yo jamás regalaría un hijo", entre otros. La consideración de la idea de descartarlos en lugar de donarlos llegaba a resultar violenta para algunos participantes y al menos difícil para la mayoría, con comentarios al respecto, tal como lo expresara una mujer "nunca me convertiría yo en una criminal ni ofendería yo a mi Dios con algo asî". Sobre la donación de embriones para terapia de fertilidad, las opiniones iban desde un "No, puede sonar un poco egoísta, pero no, no lo donaría. No critico a quien lo haga, pero yo no lo haría", hasta un "Sí, porque si se puede ayudar a alguien, y si yo ya no puedo tenerlos conmigo, pues mejor que estén con alguien que sí los va a poder cuidar como se debe", pasando por "Dependería de la vivencia, porque lo que yo he pasado es mucho, porque si no ha pasado nada con la otra pareja y me dicen oye dóname, no, ahí no. Supongo que debe de haber un proceso para todo eso".

Quien opinó sobre la donación de embriones con fines de investigación, comentó a favor que "Sí, porque igual es bueno, si investigaran más, tal vez habría más embarazos, porque esto no es seguro, o habría menos intentos para conseguir el embarazo. Nosotros llevamos tres, pero hemos conversado con parejas que han hecho hasta ocho intentos y debe ser más duro, hasta en lo económico, porque todo esto es caro, no está al alcance de todos" (hombre) o "Si sé que la investigación va a ayudar a parejas que tiene una problemática similar a la mía, sí, pero tendría que asegurarme de alguna manera" (mujer). Comentarios en contra iban en el sentido de decir, por ejemplo, que "No, porque investigar por investigar sería como hobbie, o que pudieran modificar su propia naturaleza de fabricar superhombres, o hacer cosas raras, que lo alteren a que sea otra cosa que no es, no, no, ahí si no" (hombre) o "Qué fuerte pregunta (silencio). No lo había pensado jamás (silencio). O sea, el niño no va a nacer, porque si se va a trabajar en un laboratorio, pues no nace. Pero... tal vez lo donaría. No lo había pensado" (mujer), "No creo, no, suena horrible, no sé si se pueda, pero suena feo que en lugar de un bebe salga otra cosa" (mujer).

De diferentes modos se puede dejar entrever que el cristianismo, por un lado, y las interpretaciones personalistas del origen de la vida humana, por otro lado, se leen entre líneas en los comentarios de varios entrevistados. La equiparación del estatus ontológico del embrión con un hijo es una de las interpretaciones más comunes.

Cabe destacar que no existe legislación especial respecto a la reproducción asistida. Parece ser que el problema de asincronía entre ciencia y ley es generalizado, desde naciones desarrolladas a otras menos desarrolladas. Los adelantos científicos y la práctica de estos no se han acomodado del todo al ámbito del derecho en sitio alguno. Existe en ese sentido un vacío legal. La práctica de las TRA es legal en el Perú por varias razones. Primero, se trata de un derecho humano fundamental 
recogido en la Constitución. El derecho a la paternidad es un derecho humano. En la Constitución peruana se plasma el derecho a la paternidad responsable en el Capítulo II, "De los derechos sociales y económicos", en el Artículo 6, el cual dice que "La política nacional de población tiene como objetivo difundir y promover la paternidad y maternidad responsables. Reconoce el derecho de las familias y de las personas a decidir." La Constitución protege a la familia, unión de hecho y matrimonio. No es delito la aplicación de las TRA, porque tendría que estar tipificado en una ley sancionadora (Código Penal; de acuerdo con el Principio de legitimidad, no hay delito sin ley previa). Al no estar específicamente regulados, la filosofía del derecho comúnmente estima que debe encuadrarse dentro de la moral, las buenas costumbres y no ser contrarias al orden público.

Para el caso de la donación y recepción de gametos, existe la Ley 28189, "Ley general de donación y trasplante de órganos y/o tejidos humanos", a la cual es posible adscribirse sin ningún problema, prevaleciendo los principios de altruismo, solidaridad, gratuidad, anonimato y ausencia de lucro.

Algo más controvertido es lo relacionado con la gestación surrogada. La Ley General de Salud (26842), Título I "De los derechos, deberes y responsabilidades concernientes a la salud individual", Artículo 7, dice que "Toda persona tiene derecho a recurrir al tratamiento de su infertilidad, así como a procrear mediante el uso de técnicas de reproducción asistida, siempre que la condición de madre genética y de madre gestante recaiga sobre la misma persona. Para la aplicación de técnicas de reproducción asistida, se requiere del consentimiento previo y por escrito de los padres biológicos". En este sentido, se ha llegado a juzgar como discriminatorio que la condición de madre genética y gestante recaiga en la misma mujer ${ }^{(27)}$. Sin embargo, en caso de necesitar de una gestación surrogada, al estar contenida en una ley superior (la Carta Magna, la Constitución, Ley de Leyes), debe atenerse a la superior antes que a la inferior. Por ello, es posible la gestación surrogada en el Perú, por el rango Constitucional que adquiere, además de otros factores, con la salvedad de esta discordancia en la Ley 26842. Los requisitos para solicitar este tipo de tratamientos serían la decisión libre, consciente y voluntaria, así como que existan razonables posibilidades de éxito, habiéndose descartado las posibilidades fisiológicas para poder procrear.
Además, existe la Ley 28457, la cual "Regula el proceso de filiación extrajudicial de paternidad extramatrimonial”. Esta ley incorpora la prueba de ADN como de alta certeza para que el juez declare la paternidad, lo cual tiene repercusiones en la gestación surrogada, ya que faculta al médico a otorgar un certificado sin ir contra la verdad ni contra la ley de quiénes son los padres genéticos del niño. El último dato a anexar respecto a la gestación surrogada es la jurisprudencia ya asentada por una sala ejecutoria del Callao, a través del expediente No 4568-2002-8vo JPC, donde establece que la existencia de un vacío legal en reproducción asistida no es delito, además de que convalida la expedición del certiffcado de madre genética (así esta no tenga condición de madre biológica), debiendo imperar siempre en esta cuestiones (como en todo el derecho que tiene que ver con menores) el interés superior del menor.

Por otro lado, también resulta interesante que los profesionales de la medicina reproductiva en América Latina parecieran coincidir en parte con las afirmaciones de los pacientes entrevistados. La Red LARA se reunió, en 1995, en Reñaca, Chile, para trabajar sobre un "Consenso latinoamericano en aspectos ético-legales relativos a las técnicas de reproducción asistida", publicado al año siguiente. En este documento se indica que "La comunidad científica latinoamericana no intentó definir los inicios biológicos de una persona; de hecho, no es a través de la biología que se llega a esta definición. A pesar de ello, existe consenso que el camino a ser persona se inicia una vez completada la fertilización y establecida la individualidad genética" (28). Por este motivo es que varios hospitales adheridos a la Red LARA criopreservan células en estado de pronúcleos (como el caso de Chile), al considerar que aún no se encuentran ante ese estado de "camino a ser persona", por no haber ocurrido la primera división mitótica. Además, el documento señala que "Todo programa de criopreservación debe ir ligado a un programa de donación o adopción de productos de la concepción que permita encontrar una madre para aquellos productos que no serán transferidos a sus progenitoras". De esta manera, es entendible que la red haya generado modelos de consentimiento informado, donde se considere esta situación. En el texto propuesto para la "criopreservación de células en estado de pronúcleo" dice "En caso de decidir no hacer uso de estas células en estado de pronúcleos o embriones, en un plazo de 3 años, desde la criopreservación y habiéndolo manifestado por escrito, la pareja debe autorizar a la Unidad de Medicina Reproductiva, para donarlas en forma confidencial y anónima a parejas infértiles que deseen recibirlas en adopción. Las células en estado de pronúcleo no serán destruidas" (29). Estas observaciones resultan importantes, ya que por un lado llama la atención la carga psicológica y cultural que puede venir con la palabra 'adopción', al referirse a un embrión, y por otro lado que durante la entrevista no parecía que estos textos hubiesen influenciado las opiniones de los participantes, sino que más bien parecía que coincidían en parte con las opiniones de ellos. Además, la distinción que la Red hace entre células en estado de pronúcleos y el embrión no estaba presente en ninguno de los entrevistados.

El primer estudio que intenta una aproximación al tema de la donación de embriones fue realizado también en Chile, donde se exploró la ansiedad que despierta la idea de la donación hipotética de embriones con fines de investigación, encontrándose que los tipos de ansiedad más frecuentemente asociados fueron la ansiedad de culpa, de separación y de mutilación ${ }^{(30)}$. Esto muestra que el proceso psicológico de enfrentar esta situación es complicado; en parte, por creencias donde se 'personaliza' al embrión. Posteriormente, un estudio similar encontró que los tipos de ansiedad más comunes en la población peruana fueron la ansiedad de culpa, la ansiedad de separación y la ansiedad de vergüenza ${ }^{(31)}$. Con un análisis desde la perspectiva de género, se sugirió que algunas diferencias en la ansiedad generada dependían de factores asociados a la construcción de las masculinidades y las feminidades en los participantes entrevistados ${ }^{(32)}$. Estos estudios, junto a reflexiones teóricas, llevaron a proponer la necesidad de generar políticas públicas explícitas en relación con la investigación con embriones humanos ${ }^{(33)}$, terreno aún menos explorado en América Latina.

Finalmente, del grupo estudiado, se puede comentar que la donación de gametos es un tema más comentado y generalmente aceptado por los participantes; la donación de embriones es un tema menos discutido (por falta de información en muchas de las ocasiones) y más conflictivo al momento de pensar acerca de él, para recibirlos y aún más para donarlos. La criopreservación es un tema complejo, comentado pero también muy conflictivo, cuya aceptación o no, así como el destino posible de los embriones 
probablemente criopreservados, depende de las concepciones que tienen los participantes respecto al origen de la vida (en muchos casos influenciadas con ideas personalistas), su ética personal, su construcción del género y su forma personal de vivir su religión (ya que las opiniones personales vertidas en las entrevistas no necesariamente reflejan las posturas oficiales de la religión con la cual se identificaban o decían practicar). Con todo lo anterior, se puede plantear que las hipótesis que pueden construirse desde el presente estudio cualitativo, para poder ser contrastadas en un futuro con una muestra estadísticamente representativa de esta población y así sugerir generalizaciones, serían que la donación de embriones podría efectuarse, principalmente, con fines de terapia de fertilidad y excepcionalmente con fines de investigación. La importancia de verificar esto en el futuro es, como se hiciera notar previamente, que en la mayoría de estudios disponibles realizados en países desarrollados la donación con fines de terapia de fertilidad es minoritaria, mientras que descartar los embriones o donarlos para investigación y/o experimentación es predominante.

\section{AGRADECIMIENTOS}

El autor es becario del Consejo Nacional de Ciencia y Tecnología (CONACYT), México.

El autor es Premio en Bioética 'Manuel Velasco-Suárez 2007' PAHEF-PAHO/WHO y el único responsable por los puntos de vista expresados, los cuales no necesariamente reflejan la opinión o política de la Pan American Health and Education Foundation o de la Pan American Health Association.

Durante la elaboración del presente trabajo no hubo conflicto de intereses.

\section{REFERENCIAS BIBLIOGRÁFICAS}

1. Fathalla MF, Rosenfield A, Indriso C, Sen DK, Ratman SS. Salud reproductiva: problemas mundiales. En: Rosenfield A, Fathalla F, editores. Manual de Reproducción Humana (FIGO). Madrid: The Parthenon Publishing Group; 1994. p. 38699.

2. Vargas L, Seminario J, García M. Experiencia con inseminación intrauterina. Ginecol Obstet (Perú). 1999;43(1):57-9.

3. Armas $S$, Rechkemmer A, García M. Inseminación intrauterina en mujeres infértiles atendidas en el Hospital Nacional Arzobispo Loayza (1995-1997). Rev Med Hered. 1999;10(1):19-27.

4. Steptoe PC, Edwards RG. Birth after implantation of a human embryo. Lancet. 1978;2(8085):366.

5. Costoya AA, Schmitt YJM, Rey GM, Dujoune CS, Sánchez UMI, Godan MA y col. Embarazo obtenido por fertilización "in vitro" y transferencia embrionaria. Rev Chil Obstet Ginecol. 1984;49(3):206-16.

6. Red Latino Americana de Reproducción Asistida. Registro Latinoamericano de Reproducción Asistida. Santiago de Chile: Red LARA; 1999, 2000, 2001, 2002, 2003. Disponibles en: http://www. redlara.com

7. Trounson A, Mohr L. Human pregnancy following cryopreservation, thawing and transfer of an eight cell embryo. Nature. 1983;305(5936):707-9.

8. Cruz-Coke Madrid R. ¿Desde qué momento hay alma en el embrión humano? Rev Med Chile. 2002;130(5):590-2.

9. Salinas PH, Adauy EA, Ide VR. Reflexiones acerca de células troncales obtenidas mediante la utilización de embriones humanos. Rev Chil Obstet Ginecol. 2005;70(4):264-71.

10. The Practice Committee of the American Society for Reproductive Medicine and the Practice Committee of the Society for Assisted Reproductive Technology. 2006 Guidelines for gamete and embryo donation. Fertil Steril. 2006;86(Suppl 5): S38-S50.

11.ESHRE Task Force on Ethics and Law. III. Gamete and embryo donation. Hum Reprod. 2002;17(5):1407-8.

12.Zegers-Hochschild F. Attitudes towards reproduction in Latin America. Teachings from the use of modern reproductive technologies. Hum Reprod Update. 1999;5(1):21-5.

13. Van Voorhis BJ, Grinstead DM, Sparks AE, Gerard $J$, Weir RF. Establishment of a successful donor embryo program: medical, ethical, and policy issues. Fertil Steril. 1999;71(4):604-8.

14. Lyerly AD, Faden RR. Willingness to donate frozen embryos for stem cell research. Science. 2007;317:46-7.

15. Kovacs GT, Breheny SA, Dear MJ. Embryo donation at an Australian university in-vitro fertilisation clinic: issues and outcomes. Med J Aust. 2003;178(3):127-9.

16. McMahon CA, Gibson FL, Leslie Gl, Saunders DM, Porter KA, Tennant CC. Embryo donation for medical research: attitudes and concerns of potential donors. Hum Reprod. 2003;18(4):871-7.

17. Burton PJ, Sanders K. Patient attitudes to donation of embryos for research in Western Australia. Med J Aust. 2004;180(11):559-61.

18. Fuscaldo G, Savulescu J. Spare embryos: 3000 reasons to rethink the significance of genetic relatedness. Reprod Biomed Online. 2005;10(2):164-8.

19. Cattoli M, Borini A, Bonu MA. Fate of stored embryos: our 10 years experience. Eur J Obstet Gynecol Reprod Biol. 2004;115(Suppl 1):S16-8.

20. Hammarberg K, Tinney L. Deciding the fate of supernumerary frozen embryos: a survey of couples' decisions and the factors influencing their choice. Fertil Steril. 2006;86(1):86-91.

21. Bangsboll S, Pinborg A, Yding Andersen C, Nyboe Andersen A. Patients' attitudes towards donation of surplus cryopreserved embryos for treatment or research. Hum Reprod. 2004;19(10):2415-9.
22. Newton CR, McDermid A, Tekpetey F, Tummon IS. Embryo donation: attitudes toward donation procedures and factors predicting willingness to donate. Hum Reprod. 2003;18(4):878-84.

23. Dyer SJ, Abrahams N, Hoffman M, van der Spuy ZM. 'Men leave me as I cannot have children': women's experiences with involuntary childlessness. Hum Reprod. 2002;17(6):1663-8.

24. Arranz L, Blum B, Ito E. Estudio de un grupo de mujeres sujetas a tratamientos de reproducción asistida: Un enfoque cualitativo. Salud Ment. 2001;24(5):30-6.

25. Lyerly A, Brelsford E, Bankowski B, Faden R, Wallach E. A qualitative study of individuals' attitudes regarding their cryopreserved embryos. Int Congr Series. 2004;1271:353-6.

26. Nachtigall RD, Becker G, Friese C, Butler A, MacDougall K. Parents' conceptualization of their frozen embryos complicates the disposition decision. Fertil Steril. 2005;84(2):431-4.

27. Roa Meggo Y. Infertilidad y nuevas tecnologías reproductivas en el Perú: un abordaje psicosocial. Rev Per Obst Enf. 2007;3(1):73-79

28. Red Latinoamericana de Reproducción Asistida. Consenso latinoamericano en aspectos éticolegales relativos a las técnicas de reproducción asistida. Cad Saúde Públ Rio de Janeiro. 1998;14(Supl 1):140-6.

29. Red Latinoamericana de Reproducción Asistida. Formularios de educación y consentimiento en procedimientos de reproducción asistida. Santiago de Chile: Red LARA; 2001. Disponible en: http:// www.redlara.com/fdash4509.asp?arq=Consenti mientos\%20informados.pdf

30. Álvarez-Díaz JA. Tipos de ansiedad de las parejas tratadas con fertilización asistida frente a la donación hipotética de embriones en el Hospital Clínico de la Universidad de Chile. Ginecol Obstet Mex. 2005;73(4):163-72.

31. Álvarez-Díaz JA. Tipos de ansiedad de las parejas tratadas con fertilización asistida frente a la donación hipotética de embriones en una clínica privada en Lima, Perú. Gac Med Mex. 2006;142(3):223-8.

32. Álvarez-Díaz JA. Tipos de ansiedad frente a la donación hipotética de embriones para investigación. Una aproximación desde la perspectiva de género. Salud Ment. 2006;29(5):59-65.

33. Álvarez-Díaz JA. Políticas públicas e investigación en embriones humanos. Rev Fac Med (Mex). 2005;48(6):242-7.

Manuscrito recibido el 10 de marzo de 2008 y aceptado para publicación el 13 de mayo de 2008.

\section{Correspondencia:}

Dr. Jorge Alberto Álvarez Díaz

Universidad Complutense de Madrid, Facultad de Medicina. Plaza de Ramón y Cajal s/n, Unidad de Historia de la Medicina, Pabellón IV, Sótano, Despacho 3. CP 28040, Madrid, España

Correo-e: bioetica_reproductiva@hotmail.com 